\title{
Incidencia de la banca en el sector agrícola primario ecuatoriano
}

\section{Incidence of banking in the ecuadorian primary agricultural sector}

Francisco Quinde Rosales

Universidad Agraria del Ecuador, Ecuador

Rina Bucaram Leverone

Universidad Agraria del Ecuador, Ecuador

Víctor Quinde Rosales

Universidad Agraria del Ecuador, Ecuador

Autor para correspondencia: panchoqr@hotmail.com; rbucaram@uagraria.edu.ec; vquinde@uagraria.edu.ec

Fecha de recepción: 10 de Octubre 2017 - Fecha de aceptación: 15 de Febrero de 2018

Resumen: El presente artículo analiza la influencia que tiene el Sector Bancario Nacional, tanto público como privado, en el desarrollo económico del sector Agrícola Primario del Ecuador. Esto a través de las asignaciones crediticias de las diferentes instituciones bancarias con que cuenta el país y el Producto Interno Bruto Agrícola durante el periodo 2005-2015. El análisis estudió las variables Crédito Privado Agrícola y Crédito Público Agrícola la cuales muestran un desarrollo muy marcado durante el periodo estudiado evidenciando el gran interés por parte de la banca en invertir en el sector agrícola primario. También se estudió el comportamiento del Producto Interno Bruto Agrícola el cual muestra un crecimiento continuo en el periodo 2005-2015. Finalmente, al aplicar el modelo de regresión múltiple en un software econométrico para el análisis en cuestión, se muestra que la variable crédito público agrícola y crédito privado agrícola en función del Producto Interno Bruto Agrícola son variables poco significativas para explicar el comportamiento del PIB Agrícola incluso se muestra una relación inversa la banca privada.

Palabras Claves: crédito público; crédito privado; pib agrícola; regresión econométrica; sector agrícola primario; desarrollo económico

Abstract: The present paper analyzes the influence that have the national banking sector, both the public and private sector, in the economic development of the primary agricultural sector of Ecuador. This through of the financial allocations of the different banking institution from the country and the gross domestic product during the period 2005-2015. The analysis studied the variables Private Agricultural Lending and Public Agricultural Lending, which indicate a very distinctive development during the studied period evidencing the huge interest by the banking system to invest in the Primary Agricultural Sector. The Agricultural Gross Domestic Product behavior was also studied, which shows a continued growth in the period 2005-2015. Finally, implementing the Liner Regression in the Eviews system for the concerned analysis, it shows that the variables Private Agricultural Lending and Public Agricultural Lending according to the Agricultural Gross Domestic Product are irrelevant variables to explain the Agricultural Gross Domestic Product behavior, and even it shows an inversely relationship. Key Words: public lending; private lending; agricultural gross domestic product; econometric regression; primary agricultural sector; economic development 


\section{Introducción}

La historia de la Republica ecuatoriana presenta una relación entre la independencia de su territorio ante el yugo español y el florecimiento del sector agrícola en términos productivos y comerciales (Naranjo, 2009). La agricultura mantiene su importancia en el desarrollo económico del país entendiendo que este no se encuentra aislado del resto de sectores. Hirschman (1958) y Arias et al. (2005) coinciden en que la agricultura sigue siendo aún el ente de desarrollo económico más importante que el del resto de sectores. Hayami et al. (1985) lo describe como la interrelación de la agricultura e independencia de la misma con el resto de la economía. La Organización de la Naciones Unidas para la Alimentación y la Agricultura - FAO (1995) complementa al decir que, para lograr el crecimiento económico de un país, la agricultura debe aportar articuladamente con el resto de sectores para maximizar el beneficio de las ventajas competitivas y acumular capital. La Comisión Económica para América Latina y el Caribe CEPAL (2001) describe la agricultura tiene una influencia en el medio de vida rural y la soberanía alimentaria y que para corregir la pobreza de sus pobladores es necesario el desarrollo de la actividad económica de los territorios rurales generando una agricultura dinámica y competitiva que genere empleo y a su vez una demanda de servicios y productos que dinamice la economía.

Echeverri et al. (2002) citado por FAO (2004) mencionan que la agricultura tiene una capacidad para abordar dimensiones económicas y no económicas que en la actualidad son prioritarias para todos, entendiendo que la agricultura genera un conjunto de externalidades las cuales pueden ocasionar beneficios o perjuicios a la actividad productiva y que se escapan de la misma pero que afectan al conjunto social. Quinde (2015) manifiesta que estos tres factores demuestran que el sector agrícola no solo es la actividad primaria productiva, el Ecuador debe tomar al sector como un activo estratégico. Yannuzzelli (2017) menciona que el sector agrícola es el eje vinculante de una economía en vía de desarrollo, por lo tanto, es menester generar financiación que conlleve al progreso de este sector.

Para conservar y mejorar a este sector es necesario establecer y promover fuentes de financiamiento que de forma recíproca contribuyan al desarrollo productivo de mencionado sector; reconociendo la funcionalidad que existe entre la agricultura y los otros sectores en especial la industria. La FAO (1996) nombra a dos fuentes de financiamiento principales en Latinoamérica, dichas fuentes son la pública y la privada. Las asignaciones crediticias dentro de una economía no desarrollada resultan de gran importancia, ya que estas son capaces de generar la innovación necesaria para potencializar el desarrollo de determinado sector dentro de un sistema económico. Clavellina (2013) expone que una economía en desarrollo debe ver con buenos ojos al crédito bancario ya que este se vuelve en un elemento esencial para el apoyo a sus actividades productivas. Honohan (2004) reconoce la existencia de un vínculo positivo entre el financiamiento y el crecimiento entendiendo que es necesario mencionado crecimiento para reducir los niveles de pobreza de una nación. Adicional a lo mencionado Pagano (1993), expresa que los intermediarios financieros incrementan la productividad generando una relación sobre el crecimiento económico.

Mckinnon (1973), Shaw (1973) y Warman et al. (1994) establecen que es necesario una liberalización financiera que genere beneficios para el desarrollo económico de un país. Algunos economistas señalan que una correcta inversión es capaz de generar un desarrollo económico, tal 
es el caso de Fry, Levine y el propio Banco Mundial quienes señalan que la banca en un factor esencial tanto en el ahorro de las familias como en la correcta asignación de créditos promoviendo así el desarrollo económico de un país (Morales et al., 2013). Incluso la muy conocida teoría Keynesiana podría ajustarse a la necesidad de que la economía real se integre al comportamiento del sistema financiero (Morales et al., 2013).

En el Ecuador, según datos de la Súper Intendencia de Bancos (2017), las asignaciones crediticias del 2015 por parte de los Bancos Privados Nacionales a todos los subsectores de la economía, aumentaron su volumen de créditos en un $201 \%$ con respecto al año 2005 y mostraron una tasa de crecimiento promedio del $13 \%$ durante el período 2005-2015. Beneficiando mayormente durante el periodo 2005-2015 a subsectores como: comercio al por mayor y al por menor, reparación de los vehículos de motor y de las motocicletas con un $36 \%$, consumo y microcrédito $8 \%$, construcción $6 \%$, elaboración de productos alimenticios, de bebidas y tabaco $6 \%$, entretenimiento, recreación y otras actividades de servicios $6 \%$, actividades financieras y de seguros $5 \%$, agricultura $4 \%$ y fabricación de productos metálicos y no metálicos 4\% (Quinde, 2017).

Por su parte las Instituciones Financieras Públicas reflejan un crecimiento en el año 2015 del $171 \%$ en el total de créditos asignados a los diferentes sectores en comparación al año 2005, con una tasa de crecimiento promedio del 14\% durante el período 2005-2015. Los subsectores que registraron una mayor participación en promedio durante el período 2005-2015 por parte de las Instituciones Financieras Públicas son: la construcción con un 18\%; administración pública y defensa, planes de seguridad social de afiliación obligatoria 14\%; ganadería y animales $12 \%$; comercio al por mayor y al por menor, reparación de los vehículos de motor y de las motocicletas 12\%; y, agricultura 10\%. Durante el período 2005-2015 el Sistema Bancario Nacional registró \$7.697.304.726,95 en montos entregados a través del crédito al sector agrícola primario del país, de este total el $81 \%$ se entregó a través de los Bancos Privados Nacionales y el $19 \%$ restante fue entregado por Instituciones Financieras Públicas. Los Bancos Privados Nacionales registraron asignaciones crediticias durante el período 2005-2015 por un monto total de \$6.223.291.510,34 al sector agrícola; mostrando en el 2015 un crecimiento del $44 \%$ con respecto al 2005 y una tasa de crecimiento promedio durante el 2005-2015 del 9\%.

Las provincias con mayor participación en promedio durante el período 2005-2015 en las asignaciones crediticias al sector agrícolas por parte del sector privado son: Pichincha 35\%, Guayas 27\%, Los Ríos 8\%, El Oro 5\%, Tungurahua 4\%, Azuay 4\%, Santo Domingo de los Tsáchilas 4\%, Manabí con un 3\% y las provincias restantes apenas suman el 10\%. Mientras que durante el período 2005-2015 el valor total de los créditos que fueron asignados al sector Agrícola por parte de las Instituciones Financieras Públicas alcanzó el monto de \$1.474.013.216,61; para el 2015 mostró un crecimiento del 294\% con respecto el 2005 con un promedio de crecimiento durante el período 2005-2015 del 23\%. Las provincias con mayor participación en la asignación de créditos al sector agrícola por parte de las instituciones públicas son Guayas con 24\%, Los Ríos 21\%, Esmeraldas 9\%, Manabí 7\%, Pichincha 6\% y el resto de provincias suman el $33 \%$ restante (Quinde, 2017). Todos estos datos que nos muestra la Súper Intendencia de Bancos del Ecuador nos reflejan el alto nivel de participación que tiene el Sistema Financiero Nacional en la economía del país, sin embargo es importante analizar que tanto influyen en el desarrollo de un determinado sector, es por esta razón que se planteó este análisis 
ya que es trascendental conocer el verdadero efecto que tienen las inversiones por parte de la banca en el desarrollo económico del sector Agrícola Primario del Ecuador.

\section{Métodos}

El artículo se estructuró con un tipo de razonamiento inductivo, se generaron pruebas econométricas que expliquen el nivel de participación que tiene el nivel de asignación de créditos en los períodos 2005-2015 en la formación del PIB Agrícola con el uso de un software econométrico, esto nos sirvió para conocer la verdadera contribución del crédito bancario en el desarrollo de la agricultura nacional que es el fin del presente documento. El proceso de desarrollo de la investigación es unimétodo con un paradigma empírico-analítico el cual permite reflejar la realidad de la forma más fiel y neutral posible. Se obtuvo un modelo de regresión lineal múltiple que sirvió para medir la incidencia de la banca en el sector agrícola primario, esto indica que el articulo experimentará con un modelo preestablecido para evaluar la relación existente entre las variables estudiadas (Duque, 2015). Para el desarrollo de este documento se generó un diseño empírico ya que se desarrolló en base a las evidencias existentes de datos de créditos tanto públicos como privados y PIB Agrícola, dichos datos de serie de tiempo trimestral con un periodo de evaluación del 2005 al 2015 se extrajeron de las páginas del Banco Central del Ecuador (2017) y Súper Intendencia de Bancos y Seguros del Ecuador (2017).

Gujarati et al. (2010) Manifiesta que el método de mínimos cuadrados ordinarios del matemático alemán Carl Friedrich Gauss, se considera como el modelo clásico o estándar de regresión lineal - MCRL, siendo este la base de la teoría econométrica, sobre la cual se generan los modelos de regresión simple y de regresión múltiple, modelo en los cuales hay más de una variable explicativa o regresora.

Generalizando la función de regresión poblacional (FRP):

$$
Y t=\beta 0+\beta 1 X 1+\beta 2 X 2+\beta 3 X 3+\mu t
$$

Para efectos de simetría notacional:

$$
Y t=\beta 1 X 1+\beta 2 X 2+\beta 3 X 3+\mu t
$$

Y es la variable dependiente, $\mathrm{X} 1, \mathrm{X} 2$ y X3 son las variables explicativas, $\beta 1, \beta 2$ y $\beta 3$ se denominan coeficiente de regresión parcial, $\mu$ es el término de perturbación estocástica, y t es la t-ésima observación por ser series de tiempo.

\section{Resultados}

El Producto Interno Bruto - PIB Agrícola presenta una pendiente positiva dentro del periodo en estudio, comportamiento opuesto al presentado por los créditos otorgados por la Banca Privada, Banca Pública y otras Financieras el cual presenta dentro del periodo en estudio un comportamiento estacionario (Figura 1). 


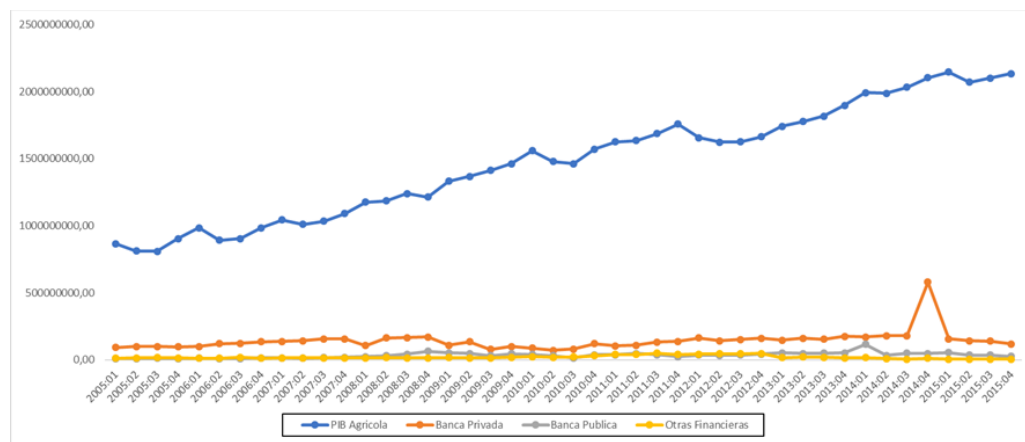

Fig. 1. PIB Agrícola, Banca Privada, Banca Pública y Otras Financieras

Para medir la relación entre el crédito bancario y el PIB Agrícola, se realizó una primera función en la cual se tomaron como variables independientes al Crédito Agrícola Público, Crédito Agrícola Privado, y el generado por otras financieras; mientras que, como variable dependiente al PIB Agrícola, obteniendo el siguiente modelo:

$$
P I B=f(\text { Banca Privada }+ \text { Banca Publica }+ \text { Otras Finacieras })
$$

Mencionadas variables presentan una simetría en la distribución de datos o una distribución normal, según lo demuestra la evaluación de normalidad generada por el diagrama de caja de bigotes (Figura 2).

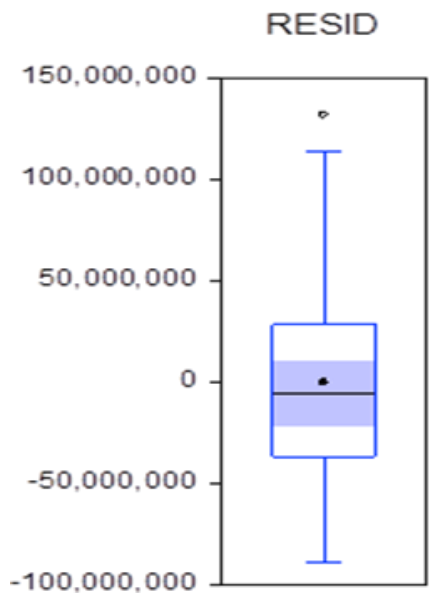

Fig. 2. Diagrama Caja de Bigotes

Para lograr una mayor cobertura en términos de la capacidad explicativa que tiene el modelo dado esto por el indicador de $\mathrm{R}^{2}$, tener una independencia de los datos según lo evalúa el test de Durbin-Watson y buscar la reducción de la probabilidad de cometer el error de tipo I evaluado por el indicador Prob (F-statistic), el modelo se modificó a variables logarítmicas de forma inicial y luego se agregó a las regresoras una variable autorregresiva de la dependiente.

$$
\begin{gathered}
L P I B=f(\text { LBanca Privada }+ \text { LBanca Publica }+ \text { LOtras Finacieras })^{1}(4) \\
L P I B=f\left(\text { LPIB }(1)^{2}+\text { LBanca Privada }+ \text { LBanca Publica }+ \text { LOtras Finacieras }\right)
\end{gathered}
$$

\footnotetext{
${ }^{1}$ La letra "L" hace alusión a la modificación de la variable convirtiéndola en logarítmica

2 Variable que presenta rezago de un periodo
} 
Cabe indicar que el modelo final favorece el resultado de los valores obtenidos en $\mathrm{R}^{2} \mathrm{y}$ Prob (F-statistic) poniéndolos a estos por encima de los valores obtenidos en los test de Akaike info criterion y Schwarz criterion (Tabla 1).

Tabla 1. Estimación de Parámetros y Pruebas Estadísticas

\begin{tabular}{lccccc}
\hline Modelo & $\mathbf{R}^{2}$ & Prob (F-statistic) & $\begin{array}{l}\text { Durbin- } \\
\text { Watson }\end{array}$ & Akaike & Schwarz \\
\hline $\begin{array}{l}\text { PIB }=f \text { (Banca Privada }+ \\
\text { Banca Publica + Otras }\end{array}$ & 0.451814 & 0.000021 & 0.637169 & 4.208 .724 & 4.224 .944 \\
$\begin{array}{l}\text { Finacieras) } \\
\text { LPIB }=\boldsymbol{f} \text { (LBanca Privada }+\end{array}$ & 0.664486 & 0.000000 & 0.612876 & -0.50404 & -0.34184 \\
$\begin{array}{l}\text { LBanca Publica + LOtras } \\
\text { Finacieras) }\end{array}$ & & & & & \\
$\begin{array}{l}\text { LPIB }=\boldsymbol{f} \text { (LPIB (1) + } \\
\text { LBanca Privada + LBanca } \\
\text { Publica + LOtras } \\
\text { Finacieras) }\end{array}$ & 0.978459 & 0.000000 & 1.899 .056 & -322.208 & -301.729 \\
\hline
\end{tabular}

La matriz de correlación parcial demuestra que no existe problema de multicolinealidad ya que las variables independientes estudiadas no están estrechamente relacionadas, desconociendo a la variable dependiente con un rezago en mencionada matriz (Tabla 2).

Tabla 2. Matriz de Correlación Parcial

\begin{tabular}{|c|c|c|c|c|}
\hline & LPIB & $\begin{array}{l}\text { LBANCA } \\
\text { PUBLICA }\end{array}$ & $\begin{array}{l}\text { LBANCOS_P } \\
\text { RIVADOS }\end{array}$ & $\begin{array}{l}\text { LOTRAS_FI } \\
\text { NANCIERAS } \\
\end{array}$ \\
\hline LPIB & 1 & & & \\
\hline LBANCA_PUBLICA & $\begin{array}{c}0.801166946835 \\
7364\end{array}$ & 1 & & \\
\hline LBANCOS_PRIVADOS & $\begin{array}{c}0.395535760034 \\
1371\end{array}$ & $\begin{array}{c}0.386976079 \\
2096476\end{array}$ & 1 & \\
\hline LOTRAS_FINANCIERAS & $\begin{array}{c}- \\
0.030871489113 \\
4712\end{array}$ & $\begin{array}{c}0.124358293 \\
6593056\end{array}$ & $\begin{array}{l}- \\
0.0959308586 \\
982624\end{array}$ & 1 \\
\hline
\end{tabular}

También se puede concluir que el modelo estudiado es homocedástico, es decir que las perturbaciones tienen la misma varianza y esta es constante para las diferentes regresoras, el test White corrobora esta aseveración (Tabla 3).

Tabla 3. Test de White

\begin{tabular}{|c|c|c|c|}
\hline \multicolumn{2}{|c|}{ Heteroskedasticity Test: White } & \multirow[b]{2}{*}{$\begin{array}{l}\text { Prob. } \\
F(14,28)\end{array}$} & \multirow[b]{2}{*}{0.1479} \\
\hline F-statistic & 1.577 .612 & & \\
\hline Obs*R-squared & 1.896 .162 & $\begin{array}{l}\text { Prob. Chi- } \\
\text { Square(14) }\end{array}$ & 0.1664 \\
\hline Scaled explained SS & 1.705 .532 & $\begin{array}{l}\text { Prob. Chi- } \\
\text { Square(14) }\end{array}$ & 0.2532 \\
\hline
\end{tabular}

En términos de autocorrelación serial podemos argumentar que el test de BreuschGodfrey Serial Correlation LM demuestra que sin la necesidad de agregar rezagos el modelo está libre de autocorrelación encontrando una explicación en el pasado (Tabla 4). Tabla 4. Breusch-Godfrey Serial Correlation LM

\begin{tabular}{lll}
\hline \multicolumn{2}{c}{ Breusch-Godfrey Serial Correlation LM Test: } & \\
\cline { 1 - 2 } F-statistic & 0.003397 Prob. F(1,37) & 0.9538 \\
Obs*R-squared & 0.003947 Prob. Chi-Square(1) & 0.9499
\end{tabular}


El test de Wald en su análisis con el estadístico F para contrastar la significatividad conjunta de los parámetros $\beta 1, \beta 2, \beta 3$ y $\beta 4$ tiene un valor 431,51 con un $p$-valor asociado de 0,00 ; por lo que al $5 \%$ rechazamos la hipótesis nula de no significancia conjunta de los parámetros (Tabla 5).

Tabla 5. Wald Test: Equation: Untitled

\begin{tabular}{ccccc}
\hline Test Statistic & Value & df & Probability \\
\hline F-statistic & 431.5180 & $(4,38)$ & 0.0000 & \\
Chi-square & \multicolumn{1}{l}{1.726 .072} & 4 & 0.0000 \\
\hline
\end{tabular}

Se establece como modelo final LPIB=f (LPIB (1) +LBanca Privada+LBanca Publica+LOtras Finacieras), el cual en términos de sustitución de coeficientes genera los siguientes resultados.

$$
\begin{gathered}
\text { LPIB }=0.991721277166 * \text { LPIB }(1)+0.00755049022349 * \text { LBANCA_PUBLICA - } \\
0.0129166979146 * \text { LBANCOS_PRIVADOS }-0.00395486572213 * \\
\text { LOTRAS_FINANCIERAS + 0.331241301218. }
\end{gathered}
$$

Al momento de analizar los resultados podemos mencionar que las variables al ser logarítmicas deben presentar una interpretación a nivel porcentual. La banca privada y otras financieras no contribuyen directamente en la formación porcentual del PIB Agrícola, mientras que la banca pública contribuye en un $0,7 \%$ a la formación porcentual del PIB Agrícola

\section{Discusión}

A pesar de los niveles de crecimiento crediticios de las instituciones públicas y privadas estas no responden de igual manera en el crecimiento del PIB Agrícola, todo esto es demostrado mediante el modelo econométrico que plantea el estudio, el cual nos muestra una relación negativa o poco significativa con respecto al PIB Agrícola. Esto se ve respaldado con el estudio titulado El Impacto del crédito en la producción del sector agrícola en Venezuela 1970- 1999, realizado por el Lic. Luis Alberto Fuentes Méndez quien señala,

El análisis de regresión efectuado para medir la influencia del impacto del crédito agrícola, tanto público como privado, sobre la producción del sector no permitió obtener valores confiables para el caso de los coeficientes de elasticidad del crédito agrícola. En la mayoría de los casos los signos resultaron ser negativos, contrarios a lo esperado, además de los bajos coeficientes de determinación de los modelos estimados (Fuentes, 2005, p.262).

Los resultados obtenidos por este estudio en particular muestran resultados similares a los de nuestro estudio el cual nos explica el hecho de que el crédito privado genera un efecto negativo en la producción y poco significativo en el caso del crédito público. Fuentes et al. (2007), llega a una conclusión similar, al manifestar que en este sentido el financiamiento otorgado por las instituciones especializadas de crédito al sector agrícola del sector público es una variable poco significativa para explicar el comportamiento de la producción agrícola en Venezuela.

De igual forma Ayala, Mena y Maravilla en su estudio titulado Contribución del Crédito Bancario al Crecimiento Económico en El Salvador, período 2004-2013, reafirma nuestra posición con respecto al crédito, al señalar, 
A través del estudio realizado se determinó que existen diversos aportes teóricos que muestran la importancia del crédito al crecimiento económico de cualquier país, aunque la mayor o menor contribución de éste depende sin duda alguna de las condiciones prevalecientes en cada una de las economías, tal es el caso de la economía salvadoreña donde la contribución del crédito productivo no ha sido significativo para impulsar el crecimiento económico del país (Ayala et al. 2014, p. 84).

\section{Conclusión}

Existe una marcada participación del crédito en su afán por lograr un mayor impulso en el sector agrícola primario del país, esto a través de las Instituciones Financieras Públicas y los Bancos Privados durante el período 2005-2015. Estas instituciones públicas y privadas han incrementado significativamente sus asignaciones crediticias al sector agrícola primario del Ecuador durante el período estudiado, logrando de esta forma establecer niveles de participación similares tanto dentro del PIB como dentro del total de créditos a todos los sectores del país.

Si bien los Bancos Privados Nacionales asignan solo el $4 \%$ del total de sus asignaciones al sector agrícola, estas superan en un $422 \%$ al total de las asignaciones agrícolas por parte de las Instituciones Financieras Públicas. Esto significa que dentro del sector agrícola los Bancos Privados Nacionales son quienes invierten más en la agricultura nacional. El promedio de participación del sector agrícola dentro de las asignaciones crediticias del sistema bancario nacional en promedio durante el período 2005-2015 alcanza un 7\% ya que los Bancos Privados Nacionales tienen el 4\% y las Instituciones Financieras Públicas el 10\%, mismo porcentaje en participación que tiene el PIB Agrícola dentro del PIB Nacional. En otras palabras, el PIB Agrícola y las asignaciones crediticias al sector agrícola participan en igual proporción dentro del PIB Nacional y las asignaciones crediticias nacionales.

El sistema financiero nacional creció en el nivel de asignaciones crediticias para el 2015 un $66 \%$ con respecto al 2005, mientras que el PIB Agrícola solo creció un 38\% con respecto al 2005. Esto significa que si bien el monto de préstamos aumento de manera significativa el PIB Agrícola no respondió de la misma forma dentro de la economía del país. Los resultados de la regresión econométrica realizada nos muestran una relación poco significativa por parte del crédito de las instituciones públicas con respecto a la producción y negativa en el caso de los bancos privados y otras financieras. Por lo tanto, se concluye que el crédito no es una variable significativa en el corto plazo dentro del proceso de producción agrícola primaria del Ecuador.

\section{Bibliografía}

Arias, J.; Vallejo, S. y Trejos, R. (2005). La Real Contribución de la Agricultura a la Economía del Ecuador. Ecuador: Instituto Interamericano de Cooperación para la Agricultura - IICA.

Ayala, D.; Mena, G. y Maravilla, F. (2014). Contribución del Crédito Bancario al Crecimiento Económico en El Salvador, período 2004-2013. San Salvador: Universidad de El Salvador

BCE, Banco Central del Ecuador. (2017). Obtenido de https://contenido.bce.fin.ec/home1/estadisticas/bolmensual/IEMensual.jsp

CEPAL, Comisión Económica para América Latina y el Caribe. (2001). Revalorar la agricultura y el desarrollo rural para la sustentabilidad. México

Clavellina, J. (2013). Crédito bancario y crecimiento económico en México. Economía Informa Duque, E. (2015). Seminario Metodología de la Investigación, Universidad Nacional de Colombia 
Echeverri, R. y Ribero, M. (2002). Nueva ruralidad: visión del territorio en América Latina y el Caribe. San José: Instituto Interamericano de Cooperación para la Agricultura - IICA

FAO, Organización de las Naciones Unidas para la Alimentación y la Agricultura. (1995). El Estado Mundial de la Agricultura y la Alimentación. Roma

FAO, Organización de las Naciones Unidas para la Alimentación y la Agricultura. (2004). Política de desarrollo agrícola. Roma

FAO, Organización de las Naciones Unidas para la Alimentación y la Agricultura. (1996). La inversión en la agricultura: evolución y perspectivas. Roma: Banco Mundial - BM. Cumbre Mundial Sobre La Alimentación

Fuentes, L. A. (2005). El impacto del crédito en la producción del sector agrícola en Venezuela, 1970 - 1999, Universidad de los Andes

Fuentes, L. y Anido, J. (2007). Impacto del Crédito en la Producción del Sector Agrícola en Venezuela, 1970-1999. Visión Gerencial. No. 1. ISSN: 1317-8822.

Gujarati, D. y Porter D. (2010). Econometría, México D.F. México, The McGraw-Hill Hayami, Y. y Vernon, R. (1985). Agricultural Development: An International Perspective.

Baltimore: Johns Hopkins University Press

Hirschman, A. (1958). The Strategy of Economic Development. New Haven: Yale University Press.

Honohan, P. (2004). Financial development, growth and poverty: how close are the links?, Policy research working paper. Banco Mundial

McKinnon, R. (1973). Money and Capital in Economic Development. Washington DC.: The American Political Science Review

Morales, F. J., García, G., \& Uribe, E. Á. (2013). Sistema financiero y actividad económica en México: negocio y divergencia del sector bancario. Análisis Económico, vol. XXVIII, núm. 67, 172-173.

Naranjo, M. (2009). Un Puerto en busca de una Nación, Guayaquil y la idea fundacional del Ecuador como país. Ecuador: Facultad Latinoamericana de Ciencias Sociales - FLACSO.

Seminario Internacional Poder, Política y Repertorios de la Movilización Social en el Ecuador Bicentenario

Pagano, M. (1993). Financial Markets and the Macroeconomy. Financial markets and growth An overview. European Economic Review

Quinde-Rosales, F. (2017). Modelo de Regresión Lineal, para medir la incidencia del sector Bancario en el sector Agrícola primario ecuatoriano Periodo 2005 - 2015.Ecuador:

Universidad Agraria del Ecuador - UAE

Quinde-Rosales, V. (2015). Efecto de la Intervención Estatal en la Regulación de los Precios del Arroz Cantón Daule. Ecuador: Universidad Agraria del Ecuador - UAE

SBS, Super Intendencia de Bancos y Seguros. (2017). Obtenido de http://estadisticas.superbancos.gob.ec/portalestadistico/portalestudios/?page_id=327

Shaw, E. (1973). Financial Deepening in Economic Development. Nueva York: Oxford University Press

Warman, F. y Thirlwall, A. (1994). Interest rate, savings, investment and growth in México, 1960 -1990: Tests of financial liberalization hypothesis, Journal of Development Studies

Yannuzzelli, J. P. (2017). El Índice de Orientación Agrícola (IOA) como Mecanismo del Análisis del Gasto Público Agropecuario Ecuatoriano. Período 2005 - 2015. Ecuador: Universidad Agraria del Ecuador - UAE 